\title{
Avis et ressentis des enseignants en éducation physique sur la méthode ObLoMoV (Obesity and Low Motility Victims)
}

\author{
Maurine Remacle, Nicolas Franck, Alexandre Mouton et Marc Cloes
}

\author{
Maurine Remacle. \\ Nicolas Franck. \\ Alexandre Mouton. \\ Marc Cloes.
}

Résumé. L'approche ObLoMoV vise à susciter l'intérêt envers la pratique d'activité physique de jeunes physiquement déconditionnés, en s'appuyant sur la combinaison du High Intensity Interval Training (entraînement par intervalles à haute intensité) et la théâtralisation. L'objectif de cette étude consiste à analyser les avis et les ressentis des enseignants en éducation physique (EP) qui ont testé cette approche. Cinq enseignants ont suivi une formation de deux jours visant à expliquer les principes de la méthode et à leur permettre d'acquérir des compétences fondamentales liées à l'utilisation de techniques de dramatisation. Ensuite, ils ont proposé un cycle de dix séances à leurs élèves. Des questionnaires et/ou entretiens ont été proposés avant et après la formation, avant et après le cycle. Les enseignants ont envoyé des rapports audio après chaque leçon. De manière globale, les enseignants sont satisfaits de cette méthode mais quelques améliorations sont nécessaires pour proposer le projet à d'autres intervenants.

Mots-clés. Activité physique, Santé, Éducation physique, High Intensity Interval Training, Jeu

\section{Introduction}

Dans la société actuelle, les enfants sont de plus en plus incités à adopter un mode de vie sédentaire. En effet, le développement de certaines technologies comme les jeux vidéo, les tablettes, les ordinateurs, les réseaux sociaux ... n'aident pas les jeunes à opter pour un mode de vie sain et actif (Simon, Klein \& Wagner, 2005). Morris (2013) met également en avant que les enfants inactifs deviennent, dans la plupart des cas, des parents inactifs, ce qui engendre un cercle vicieux duquel il est difficile de sortir. Parallèlement, l'obésité est également un problème de plus en plus fréquent chez les jeunes. A nouveau, le développement de la restauration rapide, la publicité pour les snacks et autres friandises n'encouragent pas les jeunes à manger sainement (Werle, 2014). A la vue de ces constats alarmants, un projet a été élaboré et a bénéficié du soutien de la Commission européenne. Il est nommé ObLoMoV. L’objectif de ce projet consiste à expérimenter une approche originale d'intervention éducative et sociale pour encourager un style de vie sain et actif chez les préadolescents (11-13 ans). Cette approche se distingue des autres par la combinaison d'exercices de High Intensity Interval Training (HIIT) et de techniques théâtrales, le but étant de plonger les jeunes dans un contexte imaginaire pour les rendre plus actifs.

L'étude présentée dans cette publication a pour objectif d'analyser les avis et les ressentis des enseignants en éducation physique (EP) vis-à-vis de l'approche ObLoMoV. Cette recherche a été développée dans le cadre d'un programme Erasmus + dont l'objectif consiste à analyser la mise en place du projet ObLoMoV dans l'enseignement primaire en Belgique.

\section{Méthodologie}

Avant de mettre en place le projet ObLoMoV en Belgique francophone et afin que celui-ci réponde au mieux à la culture du pays et aux nouvelles réformes (Pacte pour un Enseignement d'Excellence, 2017), nous avons proposé trois adaptations par rapport au projet initial présenté aux différents pays partenaires (Italie, Grèce, Slovénie, Finlande, 
Avis et ressentis des enseignants en éducation physique sur la méthode ObLoMoV (Obesity...

Belgique) lors de plusieurs séminaires à Milan.

1. Intégration de messages éducatifs en matière de santé. L'école et le professeur d'EP ont un rôle à jouer dans l'éducation à la santé de l'élèves (Cale \& Harris, 2006). Afin d'aider les enseignants à répondre à ces nouvelles missions, nous avons décidé d'inclure des messages éducatifs en matière de santé dans chaque séance : hydratation, alimentation, gestion du stress, sommeil, sédentarité, l'activité physique et l'ergonomie du dos.

2. Création de nouveaux scénarios. Au départ, trois scénarios ont été élaborés (Harry Potter, Pirates des Caraïbes et Peter Pan). Après en avoir fait une analyse critique, nous avons décidé de proposer d'autres scénarios basés sur des émissions télévisées connues des préadolescents belge (National Geographic, Eurosport, 30 secondes chrono, Koh-Lanta, Ninja Warrior, Fort Boyard, les Simpson).

3. Utilisation d'outils numériques et des nouvelles technologies. Il est nécessaire que les enfants découvrent les nouvelles technologies et apprennent à les utiliser à bon escient (Pacte pour un Enseignement d'Excellence, 2017). C'est dans cette perspective que nous avons programmé une activité originale. Lors des deux dernières séances, les élèves, par groupe, créent leur propre histoire comprenant des exercices de HIIT et un contexte imaginaire. Ensuite, un montage vidéo est réalisé afin de rassembler toutes les productions des élèves (Cloes, Cloes \& Cloes, 2014).

\section{Participants}

Cinq enseignants en éducation physique (EP) de l'enseignement primaire, sélectionnés sur base volontaire, ont participé à l'ensemble de l'étude. Deux femmes (E3, E4) et trois hommes (E1, E2, E5). L'ancienneté professionnelle varie : 1 an (E2), 7 ans (E1, E4), 23 ans (E5) et 25 ans (E3). Celle-ci a toute son intérêt dans le projet car les enseignants qui ont accumulé de 7 à 25 années d'ancienneté se remettraient plus facilement en question et rechercheraient de nouveaux défis et de nouvelles méthodes pédagogiques (Huberman, Grounauer \& Marti, 1989).

\section{Intervention}

Tout d'abord, sept enseignants en EP ont reçu une formation qui s'est déroulée en deux jours. Durant cette formation, les enseignants en EP ont pu tester, modifier et valider les dix séances proposées par l'équipe de l'ULiège. Lors de la formation, les professeurs d'EP ont reçu un classeur contenant les grandes lignes directrices du projet, les plans de toutes les séances, ainsi que tous les supports nécessaires à la mise en place. Tout a été conçu pour faciliter la tâche des enseignants. Ensuite, ceux-ci ont mis en place le cycle comprenant 10 séances de 60 minutes effectives. L'ensemble des séances proposées se trouve sur le site internet du projet (https://www.oblomovproject.eu/fr/ecole/).

\section{Récolte et traitement des données}

Au niveau de la formation, des questionnaires ont été remplis avant (T0) et après (T1) le séminaire. Ceux-ci avaient pour objectif d'une part, de connaitre l'avis des enseignants sur les particularités de l'approche et ainsi le comparer avant et après la formation. D'autre part, d'identifier leurs ressentis sur la formation reçue et leurs attentes et appréhensions par rapport au cycle. A la fin de la formation, un focus group a également été réalisé pour que les enseignants puissent une nouvelle fois partager et donner leur avis sur cette dernière. Au niveau du cycle, des entretiens ont été réalisés avant la première leçon (T2) et après la dernière (T3). De plus, pendant le cycle, les enseignants nous envoyaient, par courrier électronique, un compte rendu de la leçon qu'ils venaient de proposer. En parallèle, des observations informelles ont régulièrement été réalisées par les quatre auteurs. 
Actes de la 11ème Biennale de l'ARIS : Former des citoyens physiquement éduqués. Axe 1 - Ecole en santé, 10.25518/ sepaps20.268

Les données récoltées dans le cadre de ce travail sont exclusivement qualitatives. En ce qui concerne la formation, elles ont été encodées, en fonction des catégories identifiées de manière déductive. En ce qui concerne les données relatives aux entretiens de pré-cycle et de post-cycle et les journaux de bord, le traitement des données a été effectué par analyse de contenu : (1) retranscription ; (2) identification des unités de sens ; (3) codes et méta-codes.

\section{Résultats et discussion}

Dans cette partie, nous nous centrerons sur une synthèse des principaux résultats issus de ce projet.

\section{Formation}

Avant d'entamer la formation, les enseignants avaient quelques attentes par rapport à celle-ci. Certains enseignants mettent en avant leur envie d'en apprendre davantage et d'augmenter leurs connaissances (E1, E2, E3). D'autres mentionnent leur envie de découvrir un nouveau projet et une nouvelle approche pédagogique (E1, E2). L’approche particulière et la nouveauté de ce projet sont particulièrement soulignées.

Après la formation, la majorité des enseignants reste sceptique face à l'utilisation du langage imagé avec des élèves de $5^{\text {ème }}$ et $6^{\text {ème }}$ primaire (E1, E2, E4). Ceci peut s'expliquer par le fait que nous n'avons pas montré une séance type dans de réelles conditions. Les enseignants n'ont donc pas pu observer la réaction des élèves. Néanmoins, E3 et E5 mentionnent que leur avis a changé et qu'ils identifient davantage les points positifs de cette méthode. En ce qui concerne le fait de proposer du HIIT dans le cours d'EP, les avis sont tous positifs après la formation. Les enseignants paraissent convaincus du bien-fondé de la méthode et de l'intérêt de proposer ce type d'entrainement. Ils apprécient notamment le fait que chaque enfant, quel que soit son niveau physique, puisse réaliser l'effort demandé. Cependant, il est intéressant de noter que les enseignants sont plus dans l'idée que le HIIT peut avoir un impact positif sur la condition physique (E1, E2, E5) mais négatif sur la motivation des élèves (E2, E3, E4). En effet, plusieurs professeurs ont mentionné dans les questionnaires ou dans le focus group que ce type de séance pouvait s'avérer lassant à force d'être répété à 10 reprises. En ce qui concerne les messages éducatifs en matière de santé, les cinq enseignants sont tous d'accord pour dire qu'ils envisagent de les proposer en dehors du projet ObLoMoV. En analysant les scores moyens présents dans le tableau 1, la formation accroît le rôle du professeur d'éducation physique sur l'éducation à la santé de l'élève (McKenzie \& Lounsbery, 2014).

Tableau 1 : Scores moyens des représentations des enseignants par rapport aux messages éducatifs en matière de santé (/5)

\begin{tabular}{lr}
\hline Scores moyens & T1 \\
Impact des animations santé en dehors du contexte scolaire & 3,25 \\
Intérêt des animations santé dans le cours d'EP & 3 \\
\hline
\end{tabular}

La formation a été appréciée par l'ensemble des enseignants. Ils ont aimé le principe de co-construction, le fait d'avoir leur mot à dire sur les séances et de pouvoir les analyser avec les autres participants. Ils ont souligné que les séances étaient bien construites. Néanmoins, les enseignants en EP auraient aimé pouvoir tester une séance ObLoMoV dans des conditions réelles. 
Avis et ressentis des enseignants en éducation physique sur la méthode ObLoMoV (Obesity...

\section{Mise en place du cycle}

Concentrons-nous tout d'abord sur l'approche théâtrale du projet. À la question « Votre avis a-t-il changé par rapport au début du cycle ?", l'ensemble des enseignants répond de manière très positive, comme le confirme E4 : "Un grand oui. J'avais peur avec des 5 e et 6e primaires que ça ne marche pas mais finalement ça a bien marché. Ils ont bien aimé » (entretien post-cycle de E4, lignes 108-110). E1 souligne que son avis a changé quand il a vu l'implication des élèves durant les séances de productions personnelles comme le montre ce témoignage : «Le fait des dernières séances quand eux ont préparé et qu'ils se sont donnés à fond ça m’a un peu soulagé de voir qu'ils se soient autant amusés à produire leur séance. Donc oui, mon avis a changé vers la fin, c'était plus positif» (entretien post-cycle E1, lignes 131-133). L'avis sur les scénarios a donc positivement changé durant le cycle. L'enthousiasme des élèves durant les séances et les productions personnelles a fait pencher la balance en faveur de l'imaginaire. Cependant, E2 reste assez sceptique par rapport à cette approche. De nombreuses difficultés par rapport aux scénarios sont ressorties dans les journaux de bord. Au-delà de ces difficultés, E2 mentionne également des problèmes de comportement chez ses élèves et une baisse de soutien de la part de ses collègues. De plus, il est nécessaire de rappeler que E2 débute dans l'enseignement et comme l'indique Vamos (2007) les jeunes enseignants éprouvent certaines difficultés à faire le pont entre les savoirs théoriques appris durant leurs études et la réalité de terrain. Ceci peut donc expliquer le fait que E2 a peu apprécié la mise en place du cycle.

Deuxièmement, en ce qui concerne le HIIT, l'ensemble des enseignants semble satisfait de cette approche. Cependant, E1, E3 et E4 mentionnent deux difficultés majeures. La première concerne l'exécution des exercices. Les enseignants ont parfois éprouvé des difficultés pour corriger tout le monde pendant les 30 secondes. E3 pointe un deuxième problème : la gestion du chronométrage. Elle explique qu'il est compliqué de s'occuper à la fois du chronométrage, de la correction de l'exécution des exercices et tout en jouant un personnage.

Par ailleurs, en ce qui concerne les messages éducatifs en matière de santé, les enseignants estiment ne pas avoir changé d'avis sur leur intérêt, celui-ci étant toujours très positif. E1 réagit en expliquant : « Ça reste super positif car on amène quelque chose en plus en fin de séance. Les faire réagir sur leur façon de fonctionner dans la vie de tous les jours, sur leur santé» (entretien post-cycle de E1, lignes 171-173). Tous les intervenants se sont montrés convaincus de l'utilité de cette composante santé et ont affirmé vouloir réutiliser ce concept.

Enfin, en ce qui concerne les productions personnelles des élèves, tous les enseignants s'accordent une fois de plus sur le fait que leurs avis n'ont pas changé, ceux-ci s'avérant toujours positifs.

De manière globale, E3, E4 et E5 ont remarqué des changements positifs au niveau du comportement des élèves. Certains élèves plus timides se sont mis en avant comme l'explique E3 : "Je pense notamment à X qui n'est pas très bonne en sport, elle s'est bien impliquée dans la vidéo. Elle parle bien, c'est elle qui commence, elle s'est un peu valorisée là-dedans. » (entretien pos-cycle de E3, lignes 196-198). E5 a aperçu des changements au niveau santé : " Le premier impact que j'ai vu, c'est qu'il y en a qui prennent leur bouteille d'eau pour venir au cours. " (entretien post-cycle de E5, ligne 307-308). Une des titulaires responsables ajoute que ces mêmes élèves étaient plus motivés pour aller au cours d'EP. E1 est un peu plus nuancé car, par moments, les séances ont été difficiles et les élèves étaient assez turbulents. Néanmoins, il remarque un changement positif lors des productions personnelles : "Maintenant, un changement positif, c'est lors de leur production personnelle, là il y a eu un réel engagement. Même les plus perturbateurs se sont impliqués. » (entretien post-cycle de E1, lignes 212-214).

\section{Conclusion}

L'objectif principal de cette étude était d'analyser les ressentis et les avis des enseignants par rapport à la mise en place du cycle ObLoMoV dans leurs cours d'EP. Au vu des résultats obtenus, nous pouvons affirmer que tous semblent adopter 
Actes de la 11ème Biennale de l'ARIS : Former des citoyens physiquement éduqués. Axe 1 - Ecole en santé, 10.25518/ sepaps20.268

cette nouvelle approche pédagogique, avec succès. Même si certains enseignants ne sont pas encore tout à fait à l'aise avec la théâtralisation, ils sont plutôt favorables à ce concept et à l'idée de changer leurs habitudes. Les nombreuses adaptations effectuées par l'équipe belge se sont également montrées porteuses. En effet, les messages éducatifs en matière de santé ont eu un impact considérable sur les élèves mais aussi sur les enseignants, qui ont pris conscience de leur importance mais également de leur rôle dans la promotion d'un mode de vie sain et actif chez leurs élèves. Les productions réalisées par les élèves sont un aspect adopté, avec enthousiasme, par les enseignants et la direction. Ce concept a permis de renforcer de nombreuses compétences chez les élèves : créativité, coopération et solidarité. Mais aussi de valoriser les élèves auprès de leur professeur, amenant à une relation différente entre l'enseignant et eux. Néanmoins, si nous voulons que la méthode ObLoMoV perdure, quelques adaptations sont nécessaires : (1) proposer lors de la formation une séance «test » dans des conditions réelles ; (2) renforcer l'approche théâtrale au niveau de la formation ; (3) adapter les open scénarios par l'enseignant en EP en fonction des centres d'intérêt des élèves ; (4) organiser des cycles plus courts et mettre de la théâtralisation dans chaque cycle et; (5) sensibiliser également les parents, l'entourage de l'élève aux messages éducatifs en matière de santé.

\section{Bibliographie}

Cale, L., \& Harris, J. (2006). School-based physical activity interventions: effectiveness, trends, issues, implications and recommendations for practice. Sport, Education and Society, 11(4), 401-420.

Cloes, S., Cloes, A.-M., \& Cloes, M. (2014). Analyse du comportement et des attitudes d'enfants de primaire impliqués dans des pauses d'activité physique scolaires (PAPS). Sciences \& Sport, 29, Hors-série 1, S26. https://doi.org/10.1016/ j.scispo.2014.08.051

Huberman, M.-A., Grounauer, M.-M., \& Marti, J. (1989). Le cycle de vie professionnelle des enseignants secondaires : Résumé d'une recherche démentielle. Genève : Université de Genève, Faculté de psychologie et des sciences de l'éducation.

McKenzie, T., \& Lounsbery, M. (2014). The Pill Not Taken: Revisiting Physical Education Teacher Effectiveness in a Public Health Context. Research Quarterly for Exercise and Sport, 83(3), 287-292. http://doi.org/10.1080/ $\underline{02701367.2014 .931203}$

Morris, C. (2013). Designed to Move: Tackling the Epidemic of Physical Inactivity. National Civic Review, 102(4), 55-56. http://doi.org/10.1002/ncr.21157

Pacte pour un enseignement d'excellence (2017). Avis $\mathrm{n}^{\circ} 3$ du Groupe central. Disponible sur : http://www.enseignement.be/index.php?page $=28280$

Simon, C., Klein, C., \& Wagner, A. (2005). La sédentarité des enfants et des adolescents, un enjeu de santé publique. Journal de Pédiatrie et de Puériculture, 18(5), 217-223

Vamos, S. (2007). Experiences of beginning health educators and changes in their high school students' health behaviors and attitudes. Health Education \& Behavior, 34(2), 376-89.

Werle, C.-O. (2014). Les biais perceptuels influençant la consommation alimentaire de l'évolution de l'obésité. Médecine des maladies métaboliques, 8(4), 449-454.

PDF automatiquement généré le 2023-04-26 13:41:46

Url de l'article : https://popups.uliege.be/sepaps20/index.php?id=268 
Avis et ressentis des enseignants en éducation physique sur la méthode ObLoMoV (Obesity...

Publié par ULiège Library en Open Access et distribué suivant les termes et les conditions de la licence CC-BY (https://creativecommons.org/licenses/by/4.0/deed.fr) 